\title{
IMAGINARIOS TERRITORIALES, ESCALAS Y UTOPÍA
}

\author{
BLANCA REBECA RAMÍREZ VELÁZQUEZ ${ }^{1}$ \\ Universidad Autónoma Metropolitana- Xochimilco - México
}

Hablar de utopía en los tiempos contemporáneos resulta paradójico. Por un lado parecería que la posmodernidad nos abrió los ojos en relación a las promesas no cumplidas y los futuros no alcanzados de la modernidad, y promovió el dejar de pensar en el mañana para ubicarnos en el presente. Pero, por el otro, con la llegada de la globalización, nuevamente se abre al mundo la promesa de resolución de la crisis y de las diferencias económicas, sociales y culturales, para ubicarnos en la búsqueda del paraíso perdido, que muchos han contemplado como posibles en diferentes momentos de la evolución de la humanidad, y ahora lo buscan en un mundo que pretende ser a la vez globalizado y sustentable.

Llama la atención el significado del término en diferentes contextos, por las similitudes que pueden tener. Si, por ejemplo, nos ubicamos en el campo de las definiciones, para la Real Academia de la Lengua Española, la palabra utopía tiene un sentido importante de territorialidad, planificación y optimismo, ya que la define como "lugar que no existe" a lo que agrega la posibilidad de ser un "plan, proyecto, doctrina o sistema optimista que aparece como irrealizable en el momento de su formulación" (2001: 2260). Pero si observamos lo que dice el diccionario Oxford de la lengua inglesa, Utopía (con mayúscula) es considerada como "un imaginario social perfecto y un sistema político", en donde lo utópico es un adjetivo que nos lleva a considerar lo "atractivo y deseable pero impracticable" (STREVENS, 1980: 948).

Desde esta perspectiva, parecería entonces que hablar de utopía es algo banal y poco productivo, en donde los imaginarios por generar corresponden a territorios que

\footnotetext{
${ }^{1}$ Professora do Departamento de Teoría y Análisis de la División de Ciencias y Artes para el Diseño, Universidad Autónoma Metropolitana-Xochimilco.bramirez@cueyatl.uam.mx
} 
no existen, ni pueden existir, a pesar del optimismo que podamos tener en nuestra mente y espíritu.

Esta postura ha proliferado en algunos pensadores que asumen la a-territorialidad de la utopía. Parecería también que nuestras prácticas como urbanistas, geógrafos, economistas, arquitectos o planificadores pierden sentido y hasta perspectiva al carecer de objetos claros, o en ocasiones poco consistentes, de transformación o bien en donde a pesar de nuestra práctica, lo deseable es solo imaginario e irrealizable.

Sin embargo, valdría la pena preguntarnos ¿hasta dónde esta necesidad de imaginarnos lo que no es, lo que no existe, lo que queremos y los territorios que deseamos, o los que no pueden ser, no es parte del pensamiento de la humanidad y de la búsqueda incesante de la felicidad no encontrada? Parecería que dar respuesta a estas preguntas me llevaría mucho más que el espacio que tengo para expresarme en este ensayo. Sin embargo, asumiendo que la categoría de utopía tiene un sesgo territorial implícito, trataré de adelantar algunas reflexiones de por dónde están algunas discusiones sobre la relación ente utopía territorio en el momento actual, y cómo se vislumbran algunas de las perspectivas que se han enfocado a resolverlo.

\section{Formas y escalas de la utopía}

En la evolución de la humanidad ha habido diferentes formas de imaginar sus entornos, sus regiones, las ciudades el campo, y por supuesto la naturaleza, las relaciones y los derechos humanos que les son propios. Cada uno de estos aspectos tiene un lugar específico no sólo en la mente de quien o quienes lo crean, sino también en los espacios en donde se concretizaron, o también, en donde no han podido materializarse. Por otro lado, estos imaginarios, al ubicarse en diferentes escalas y dimensiones del querer individual o social, podemos afirmar que están jerárquicamente articulados en niveles que se trastocan o aíslan, pero que no son independientes uno del otro. Dependiendo de quien o cómo se genere el imaginario, cada utopía se sitúa en una escala específica para materializarse o no, al mismo tiempo que puede excluir o integrar otras, o contar con niveles diferenciales de concreción real en el territorio.

Por ejemplo, la concepción de desarrollo y sus diferentes modelos no deja de tener en sí misma un dejo fuerte de utopía. Para ello, el Iluminismo del siglo XVIII contribuyó enormemente a generarla a partir de los dos ideales que incluían en su concepción: el de la emancipación humana y el de la auto-realización. Para autores como Harvey (1996) estos ideales pueden caracterizarse como vagos, ya que, la emancipación humana, incluye un amplio rango de aspectos tales como problemas sustantivos referidos a las necesidades y deseos materiales, físicos o biológicos, inseguridad social, planeación y organización del territorio pasando por formas variadas de opresión de los individuos por el Estado, privilegios o poderes de clase o de dinastías. Aquí incluye también la emancipación a través de la superstición, falsa conciencia, la religión organizada y todas las formas de creencias irracionales (ibid.: 122). 
Por su parte, la auto-realización, en la opinión del autor, es aún más confusa ya que llama a la liberación de los poderes imaginativos y creativos con el fin de emancipar a los individuos alcanzando su desarrollo humano. Esto puede realizarse a través de otras formas de producción, distribución y consumo, el arte, la ciencia o la producción cultural, la política o las leyes ( ibid.). Estos ideales del Iluminismo se materializaron en el proyecto de la modernización capitalista que suponía que su aplicación, como creencia universal en la escala planetaria, sacaría a la humanidad del atraso en que se encontraban las comunidades primitivas, y llevaría al género humano a su realización como evolución total.

La modernidad socialista tuvo también su utopía. A través de ella, la lucha contra el capitalismo y la alienación que éste genera, conduce a un imaginario que tiende a lograr la homogeneización del hombre trabajador (incluyendo por supuesto a la mujer) y al de las "fuerzas del trabajo" (HARVEY, 2000: 54). Aquí también la generación de un espacio, ocupado de forma diferente al del capitalismo, llevaría al paraíso, considerado como un territorio independiente de las fuerzas del libre mercado y de la dominación del capital.

Ambas utopías, se ubicaban en el ámbito de las reivindicaciones planetarias y en donde alcanzar las condiciones de libertad, de democracia, igualdad permiten situarlas como objetivos generales que era preciso alcanzar. Aquí se incluyen también las condiciones de los derechos humanos que instituciones globales han querido imponer como obligatorias para "todas las naciones" independientemente de la imposibilidad que tengan para implantarlas realmente en el ámbito territorial (ibid.: 105-116), o de las condiciones políticas que priven en cada uno de los Estados nación responsables de su implantación.

El mismo autor, hace una diferenciación entre los utopismo de juego espacial y los de proceso social. En los primeros son todas aquellas posibilidades de materializar utopías, refiriéndose a las ideas que sirven para expresar y explorar una amplia gama de relaciones sociales que se generan y se materializan en formas espaciales concretas, ya que excluyen la temporalidad del proceso social y la dialéctica del cambio, dándoles estabilidad social garantizada por una "forma espacial fija" (ibid.:188). Aquí incluye desde la Utopía de Tomas Moro que buscaba la armonía y la estabilidad frente al caos que vivía la sociedad inglesa de la época, (ibid.:187), hasta las diferentes materializaciones que sobre lo urbano han imaginado y concretizado urbanistas y arquitectos en la historia de las ciudades: el sueño de Le Courbusier, y otros muchos que se han hecho en los países norteños.

A ellas podemos incluir también proyectos específicos que se desarrollan en diferentes ciudades latinoamericanas como los de Ciudad Satélite en el norte de la Ciudad de México, y los megaproyectos que han materializado las transformaciones de Santa Fe en el poniente de la misma ciudad; o el de Puerto Madero en Buenos Aires, Barra en Río de Janeiro entre otros muchos que encontramos en las principales urbes del mundo. Mención especial requeriría la forma espacial que está organizando 
la lucha contra la impunidad y la inseguridad en las grandes ciudades, en donde la búsqueda de la "tolerancia cero" es una copia de los juegos espaciales implantados en ciudades desarrolladas como Nueva York y de grupos dominantes que luchan en contra de la inseguridad urbana.

En la opinión del mencionado autor, los utopismos de proceso social se expresan en términos temporales y no espaciales (ibid.: 202) ya que no tienen materialización en formas territoriales fijas, independientemente que den origen a reorganizaciones de las estructuras espaciales. El ejemplo que proporciona de este tipo de utopismos es el del libre mercado que originó el capitalismo, en donde las diferencias regionales que origina se materializaron por ejemplo, en la existencia de países ricos y pobres (ibid.: 203-208), o que se manifiestan concretamente en territorios específicos al interior de las ciudades, a pesar de que la utopía pronosticaba que con la introducción al libre mercado, éstas desaparecerían. Su escala se ubica en el ámbito de los espacios nacionales, y de la competencia del Estado, lo que les da a estas ideas un grado diferente de utopismo en relación con las de juego espacial que tienen una dimensión urbana, local o regional, o las universales, cuya escala es más general y, por lo tanto, hasta planetaria.

Por último, Harvey maneja la necesidad reconocer la utopía a una escala más micro, a la que denomina "el cuerpo" que materializa la estrategia de acumulación del sistema capitalista, a través del trabajo, integrándose desarticuladamente a las otras utopías, sean planetarias, de proceso o las de juego espacial. En ella, la lucha por un salario digno, fuera de la alineación que impone el sistema, sería el ideal imaginado y la estrategia que le permitiera a los trabajadores obtener un tiempo y un territorio que les sirviera para satisfacer sus necesidades materiales, espirituales y sociales; esta dimensión se mueve en los ámbitos de la producción y del consumo (ibid.: 141-156).

Parecería entonces que esta desarticulación de las utopías en diferentes formas y escalas ha impedido que se materialicen en una dirección articulada que permita ver la dimensión del cambio más claramente. Es evidente que a su interior ha dejado fuera otras utopías que no quedaron integradas a las del capitalismo o socialismo, al mismo tiempo que ubicaban como excluyentes formas tangibles o materiales de integrarse entre ellas. Sería importante entonces analizar cómo han evolucionado y articulado estas y otras utopías para, con ello, tratar de percibir hacia dónde ha tomado dirección la transformación contemporánea.

\section{Sustentabilidad como paradigma utópico de la globalización}

Mucho se ha argumentado que la dimensión del capitalismo pasa ahora por la globalización como proceso de libre mercado que solucionará, desde el ámbito internacional, las deficiencias que su puesta en marcha supone al interior de las naciones (RAMÍREZ, 2003). Modernistas, planificadores y críticos han insistido, de diferentes formas, en la posibilidad que implica el abrir las fronteras al comercio internacional y 
a las redes de vinculación cultural de dimensión global para solucionar las deficiencias que el capitalismo ha generado en los Estados nación. Se ubica entonces como un proceso utópico, como los ejemplificados anteriormente.

En la opinión de Benton, la critica al modelo de desarrollo globalizado pasa por dos formas (1989: 52):

1) La postura globalifóbica que se opone a la globalización y a las políticas neoliberales en que se sustenta, al que se adscriben de diferente forma y bajo diferentes perspectivas organizaciones, instituciones y académicos (RAMÍREZ, 2003: 57-60), y

2) La postura ecológica política que insiste en la necesidad de producir sólo bajo las formas que aseguren la existencia de recursos suficientes para salvaguardar las generaciones futuras (ibid:: 173-189).

Estas posturas, ubicadas a nivel de paradigma de proceso, son consideradas también como conservadoras (BENTON, 1989: 52), y podemos afirmar que son las dos visiones utópicas del momento. En el caso de las posturas ecológicas, que pasan recurrentemente por la incesante búsqueda de la llamada sustentabilidad, pueden considerarse como un discurso construido en los países del sur (REDCLIFT, 1994: 123), y que para algunos pretende ser una postura alterna a la forma de desarrollo impuesta por los norteños y por el centro.

La importancia por hablar de la sustentabilidad nos llega a principios de la década de 1980, intentando poner orden a la degradación ambiental y al posible agotamiento de los recursos naturales existentes en el mundo. Por su parte, la globalización se erige como el ideal que re-ubicará el proceso de libre mercado en el ámbito internacional, en donde las acciones dinamizarían nuevamente la economía y las acciones de desarrollo, en contraposición a las acciones nacionales por regular mercados, las economías, la cultura y las sociedades, que son en parte las responsables de las imperfecciones del sistema.

El problema entre la necesidad de un orden mundial que regule el uso, conservación y transformación de los recursos necesarios para salvaguardar el sostenimiento de las futuras generaciones, se contradice con la necesidad de los Estados nacionales que tienden a suponer y argumentar la necesidad social del desarrollo y del cambio. Una vez más, la falta de claridad en el manejo de los procesos y las escalas se desarticula ya que este discurso general implica, casi en automático, una nueva dimensión de la local que supone un aprovechamiento de los recursos por las comunidades locales, que se manifiesta en la frase que la globalización ha propagado, argumentando que permite vincular lo local con lo global.

Sin embargo, tanto el paradigma de la sustentabilidad como el de la globalización, suponen la necesidad de articular escalas de acción contra el mal uso de los recursos, no son tan novedosos como pensamos. En ese sentido se ubica la critica sobre la aparición de la escala internacional para implementar el desarrollo que ha sido ampliamente difundida por autores diversos desde muy iniciado el proceso globalizador, $\mathrm{y}$ parecería entonces que es parte de la misma evolución del sistema (RAMÍREZ, 1991: 78-92). 
Por su parte, el de la sustentabilidad, nace como resultado de un ideal imaginado por la modernidad capitalista que asumía la posibilidad de dominar a la naturaleza como parte de la necesidad de emancipación de la humanidad y al mismo tiempo como parte de su auto-realización. El Iluminismo asumió que la naturaleza estaba para usarse y que su dominación era un proyecto posible fue parte del imaginario se basó en los supuestos ideológicos del cristianismo que la suponían como pre-condición de su existencia (HARVEY, 1996: 121) y del capitalismo que asumió la posibilidad de dominar a la naturaleza. En la actualidad, su exterminio puede ser también dominado y hasta controlado.

Para lograrlo, el optimismo tecnológico del siglo XVIII se sumó a la racionalidad científica del momento que permitiría, y todavía permitirá, la generación del conocimiento sofisticado que se requerían para "manipular a la naturaleza en aras de los propósitos de la humanidad", explotarla y hasta humanizarla de acuerdo con sus propósitos (ibid.: 125). Desde esta perspectiva, en la actualidad se asume que con el uso de tecnologías verdes, de proyectos sustentables (aunque en ocasiones no se sabe realmente que significan) la conservación y preservación de la naturaleza logrará que los fines de auto-realización y de emancipación se alcancen, al asegurar la existencia de las generaciones que vienen. La utopía se traslada de la escala del proceso a la del "manejo medio ambiental" (environmental managerialism en el original) (REDCLIFT, 1994: 133) que se ubica en el ámbito de lo local.

En el medio, aparece una nueva forma de vislumbrar a la humanidad ya no como sociedad en general sino como individuo en donde la mediación económica general se pierde apareciendo el/ella y sus interpretaciones, sus dimensiones subjetivas y sus prácticas cotidianas en el territorio. De ahí que cada vez se argumenta más sobre la necesidad de recuperar procesos de corte general, como sería la biodiversidad y la conservación de especies animales y vegetales en los espacios en donde aún están presentes, y al mismo tiempo se vislumbran imaginarios en donde la casa ecológica es la alternativa para resolver los problemas de corte general. Las mediaciones de escala en donde una política coherente, implementada a partir de una planeación acorde a ella, que permitan implementar e impulsar acciones coordinadas que se ubiquen en diferentes escalas del territorio materializando la utopía, son cada vez menos tratadas y mucho menos articuladas. A ello se une la tendencia del neoliberalismo galopante que las desarticula y pone en manos del sector privado las expectativas de la transformación utópica del futuro.

La naturaleza tiene una forma diferente de ver las relaciones. En la sociedad tenemos que ver la relación con el otro que está fuera, pero con la naturaleza tenemos que ver nuestra relación con nosotros mismos pues somos parte de ella. La utopía pasaría quizá por que aprendamos nuevamente a ver a la naturaleza como una parte nuestra, hacernos internos en ella y terminar con el binomio naturaleza-humanidad que la evolución del pensamiento ha generado. Estoy hablando de la necesidad de asumir nuevamente nuestra condición natural, que implica, en parte liberarnos de la 
racionalidad que nos permite verla como algo externo.

En la concepción de Mires, el desarrollo de la Ecología ha tenido a su interior un sesgo importante de utopía en donde posturas como la de Schumacher y su planteamiento del "Small is Beatifull" o la de Ivan Ilich con su utopía de convivencia, ambas trabajando con una crítica interesante a la tecnología (MIRES, 1990: 25-32), ubican a los imaginarios en el ámbito de lo local y particular, careciendo también de vínculos y posibilidades de articulación hacia los universales y generales. Estas propuestas se ubican en la dimensión opuesta ya que asưmen que incidiendo en el manejo local de los recursos, se consumará automáticamente la global.

Las ideas de globalización, sustentabilidad, manejo de recursos naturales, proyecto de desarrollo etc. no son neutrales. Por el contrario, si lo vemos desde la forma como la humanidad ha evaluado de forma diferencial las consecuencias de la modernización y del desarrollo, de su impacto en territorios y regiones diversas, percibimos que tienen un sesgo político importante que se manifiesta cada vez más en la necesidad de la intervención por parte de autoridades públicas nacionales, internacionales y/o locales en su implementación. De ahí que la materialización de las utopías pasa también por la necesidad de instrumentar lógicas coherentes que permitan articular visiones de proceso social con utopismos de juego espacial, como llama Harvey a la materialización de acciones que fijen realmente las transformaciones en espacios diferentes, en un juego complejo de utopías diferenciales con ubicaciones en escalas, también diferentes para implementarse.

Por último, en esta utopía sustentable que incluye a la naturaleza, se deja de lado, como en las otras utopías de la modernidad, al campo. Parecería ser que este territorio carece de posibilidad utópica, y se mantiene, como en los períodos anteriores, supeditado al imaginario urbano que lo controla y lo domina. O en su defecto, el utopismo del campo se reduce al uso de transgénicos por las empresas transnacionales o a los agronegocios como único recurso que posibilita su crecimiento y la necesidad de incluirlo en los proyectos utópicos de futuro. Retomamos la pregunta que hicimos en algún momento, en el sentido de reflexionar si ¿hay algo que el campo le haya dado a la ciudad? (RAMÍREZ, 2001: 62-64), que permita recuperar este espacio excluido del pensamiento utópico. La subordinación del campo es un aspecto clave de la utopía urbana, que es el que ha prevalecido en la mente de los utopistas, desde los clásicos hasta los modernos que requiere una redimensionalización en relación a las condiciones en las que se ha llevado a cabo la relación campo-ciudad (RAMÍREZ, 2003: 49-71).

\section{Y seguimos imaginando territorios}

A pesar del desencanto del posmodernismo, de la agudización de la crisis ambiental y del incremento de los clamores en contra de los problemas que genera la globalización, parecería que sigue habiendo una insistencia desarticulada en relación a la necesidad de construir un futuro diferente, y de imaginar espacios que no son los que se están 
materializando en el presente. Preguntaríamos, ¿vale la pena seguir pensando en una utopía? Si la respuesta es afirmativa, cómo se vislumbra ésta en la actualidad?

Coincidimos con Harvey cuando argumenta que hay una "ausencia de un obvio proyecto de cambio social" y de un movimiento social o alianza vigorosa que permita avanzar tesis y planteamiento coherentes en ese sentido (HARVEY, 2000: 229). Ante ello, las propuestas son diversas. Por un lado hay un llamado de parte de algunos autores por reconocer que los procesos no llevan una dirección linear y en donde la producción del espacio bajo el capitalismo (en donde se incluye a la producción/ transformación de la naturaleza), tiene efectos contradictorios (SMITH, 1984; REDCLIFT, 1994) a los que Harvey denomina "desarrollos geográficos desiguales" (HARVEY, 2000: 95-104). Ubicarnos en este contexto posibilitaría, según su opinión, la generación de un "utopismo explícitamente espacio-temporal" en tanto que estrategia privilegiada para explorar (y por qué no, articular) alternativas emancipadoras (ibid: 211). Esto implica la generación de una red intrincada de posibilidades alternas que se ubican diferencialmente en escalas diversas, pero que pueden tocarse y relacionarse, con el fin de materializar transformaciones de proceso y espaciales.

Sin embargo, hay autores que ven la generación de esta utopía por otras vías. La de Foucault, por ejemplo, se remite específicamente a la posibilidad de reconocer las heterotropías y los espacios otros de diferencia, ahondando en la importancia de la otredad y la alteridad. Desde ahí se hace una crítica a las normas y las reglas existentes que pueden ser trastocadas y cambiadas (FOUCAULT, 1999 [1984]: 15-26 Y RAMÍREZ, 2003).

Para Massey, la imaginación de un futuro pasa por la construcción de una globalización territorializada, que asume la posibilidad de generar espacios de co-existencia en donde el reconocimiento de diferentes narrativas (imaginarios), tanto de escalas como de formas, es un elemento fundamental para lograrlo. Al respecto ella argumenta:

Si sólo hay una sola narrativa, un futuro hacia el cual todos estamos marchando (en la forma como imaginamos al mundo) hemos suprimido entonces las genuinas y potenciales multiplicidades de lo espacial. Una historia linear organiza al espacio en secuencias temporales. Una negativa a temporalizar al espacio, hace posible el reconocer nuestras historias a la multiplicidad y acepta que el futuro no está escrito todavía. (MASSEY, 1999: 42).

La dimensión política está sin duda presente en el imaginario utópico territorial de futuro, tal y como se establece en las definiciones que dimos al inicio de este ensayo. Ésta se ubica en diferentes niveles, formas y escalas, como fueron argumentadas en los apartados anteriores. Sin embargo, más que ubicar la discusión en las contradicciones que presenta el Estado nación ante los clamores del neoliberalismo, es preciso evidenciar algunas tendencias que parecen ir más allá de estos supuestos. Al respecto, posturas como la de "Transversal Politics" se hace relevante en momentos en donde el incremento a la intolerancia racial, étnica y religiosa, en todo el mundo contrastan con otras que claman por una utopía que respete a las diferencias, y en donde se 
busca resolver los antagonismos de formas más conciliadoras y no sobre la base de las intervenciones militares, la fuerza de le represión policíaca o el autoritarismo de algunos Estados.

Esta corriente parte de suponer que el mundo puede verse desde diferentes posicionamientos que contemplan diferencialmente la realidad; pero que estos reconocimientos tienen a su interior formas de incluir también a la igualdad y la homogeniedad, que permiten evidenciar diferentes valores e identidades (DAVIS, 1999: 94). En este interactuar, reconocer la multiplicidad de relaciones que se abren al encuentro y al vínculo permiten suponer posibilidades jerarquizadas de posicionamientos que en diferentes niveles se traslapan en redes formales e informales de organizaciones, alianzas, conflictos o coaliciones (ibid.: 96). La política aparece cuando se reconocen las relaciones de poder al interior de esas redes de relaciones, posibilitando así la integración de un diálogo de cooperación que permite la interacción (ibid:: 97).

En este sentido, el conflicto no se asume entre opuestos pero tampoco asume que son reconciliables. Por el contrario, habla de la necesidad de posibilitar el diálogo y la co-existencia de proyectos, en momentos en donde las formas tradicionales de hacer política lo que muestran es una gran cantidad de aspiraciones, ideales y reivindicaciones que han quedado excluidas o sometidas por la fuerza de la autoridad. Una utopía más que se agregan a las de proceso social, las de juego espacial, que se enmarca en lo político. Sin embargo, sigue siendo un clamor que resolvería sin duda situaciones complejas como la que se presentan en las realidades periféricas al desarrollo del mundo, aunadas a algunas puntuales como la de los indígenas del sur de México, en donde la utopía de los zapatistas ha tenido que implantarse ante la cerrazón de los políticos por reconocer su diferencia, su espacialidad y su temporalidad. A ésta, sin embargo, habría que agregar otras muchas utopías existentes en diferentes latitudes y las que se generen para articularlas todas en el camino.

\section{IMAGINÁRIOS TERRITORIAIS, ESCALAS E UTOPIA}

Resumo: A partir do entendimento da categoría de utopía num viés territorial, o artigo aborda o debate sobre a relação entre utopia e territorio na contemporaneidade e como se vislumbram algumas das perspectivas que tentaram resolvê-lo.

Palavras-chave: Território, Utopia, Imaginário.

\section{TERRITORIAL IMAGINARIES, SCALES AND UTOPIA}

Abstract: From the understanding of the category of utopia in a territorial sense, this article focus the debate on the relation between utopia and territory and how to understand some of the different approaches trying to resolve them.

Keywords: Territory, Utopia, Imaginary. 


\section{BIBLIOGRAFIA}

BENTON, Ted. 1989. "Marxism and Natural Limits: An Ecological Critique and Reconstruction". New Left Review, 178, noviembre/diciembre, pp. 51-86.

DAVIS, Nira Duval. 1999. "What is transversal politics'?" Soundings, a journal of politics and culture, 12, London: Soundings, pp. 94-98.

FOUCAULT, Michel. 1999(1984). "Espacios Otros". en Versión, estudios de comunicación y política, núm. 9, México: Universidad Autónoma MetropolitanaXochimilco, pp. 15-26.

HARVEY, David. 2000. Espacios de Esperanza. Madrid: Akal. 1996. Justice, Nature and the Geography of Difference, Cambridge, Blackwell.

MASSEY, Doreen. 1999. "Philosophy and politics of spatiality: some considerations". en Power-geometries and the politics of space-time, Hettner-Lecture 1998, University of Heidelberg, pp. 27-42.

MIRES, Fernando. 1990. El discurso de la naturaleza: ecología y política en América Latina. Costa Rica: Departamento Ecuménico de Investigación.

RAMÍREZ, Blanca Rebeca. 2003. Modernidad, posmodernidad, globalización y territorio: un recorrido por los campos de las teorías. México: Miguel Ángel Porrúa, Universidad Autónoma Metropolitana, Xochimilco.

. 2003. "La vieja agricultura y la nueva ruralidad: enfoques y categorías desde el urbanismo y la sociología rural". en Sociológica, año 18, núm. 51, enero abril, pp. 49-71.

. 2000. Hay algo que el campo le haya dado a la ciudad? en Ciudades, núm. 50, abril-junio, pp. 62-64.

. 1991. "Lo internacional y lo regional. Algunas reflexiones metodológicas". en Ramírez, Blanca (comp.). Nuevas tendencias en el análisis regional. México: Universidad Autónoma Metropolitana-Xochimilco, pp. 78-92.

REAL ACADEMIA ESPAÑOLA. 2001. Diccionario de la Lengua Española. España, 22a edición, 2 tomos.

REDCLIFT, Michael. 1994. "Development and the environment". Skalir, Leslie. Capitalism and development, pp.123-139.

SMITH, Neil. 1984. Uneven Development: Nature, capital and the production of Space. London: Blackwell.

STREVENS, Peter (editor), Oxford Advanced Learner's Dictionary of Current English, Oxford: Oxford University Press. 